\title{
Noninvertible minimal maps
}

\author{
by
Sergiǐ Kolyada (Kiev), L'ubomír Snoha (Banská Bystrica) and Serger Trofimchuk (Santiago and Kiev)

\begin{abstract}
For a discrete dynamical system given by a compact Hausdorff space $X$ and a continuous selfmap $f$ of $X$ the connection between minimality, invertibility and openness of $f$ is investigated. It is shown that any minimal map is feebly open, i.e., sends open sets to sets with nonempty interiors (and if it is open then it is a homeomorphism). Further, it is shown that if $f$ is minimal and $A \subseteq X$ then both $f(A)$ and $f^{-1}(A)$ share with $A$ those topological properties which describe how large a set is. Using these results it is proved that any minimal map in a compact metric space is almost one-to-one and, moreover, when restricted to a suitable invariant residual set it becomes a minimal homeomorphism. Finally, two kinds of examples of noninvertible minimal maps on the torus are given - these are obtained either as a factor or as an extension of an appropriate minimal homeomorphism of the torus.
\end{abstract}

1. Introduction. We will be concerned with a discrete dynamical system $(X ; f)$ given by a Hausdorff topological space $X$ and a continuous selfmap $f$ of $X$ (written $f \in C(X)$ ). Usually $X$ will be compact or even compact metric.

Minimality and extensions of minimal systems belong to central topics in topological dynamics (see, e.g., $[\mathrm{Au}],[\mathrm{Br}]$ and $[\mathrm{deV}]$ ). In many important examples of minimal maps, these are homeomorphisms. In the sixties J. Auslander [AG, p. 514] formulated the problem whether a continuous map of a compact metric space onto itself which is not one-to-one can be minimal. Today, of course, it is known (owing also to J. Auslander himself) that it can. A class of examples of noninvertible minimal maps on some compact metric spaces can be found in [AY, p. 186].

Interesting examples of noninvertible minimal maps are known in interval dynamics when a suitable interval map is restricted to an invariant Cantor

2000 Mathematics Subject Classification: 37B05, 54H20.

Key words and phrases: minimal dynamical system, noninvertible map, irreducible map, open map, feebly open map, factor, extension, almost one-to-one map, quotient space, upper semicontinuous decomposition, torus. 
set. In fact, it was proved in [BKNS] that unimodal Fibonacci maps have a wild attractor (which is a Cantor set) provided that the order of the critical point is sufficiently high. By [BL], the restriction of such a map to this Cantor set is minimal and by $[\mathrm{LM}]$ the preimage of any point from this Cantor set is a singleton except for the critical point of the map whose preimage consists of two points. More generally, there are unimodal maps whose restriction to a Cantor set (the $\omega$-limit set $\omega(c)$ of the critical point $c$ ) is minimal and fails to be invertible only at $k$ points, each lying in the backward orbit of $c$ (one of them is $c$ itself) and having two preimages in $\omega(c)$ (all other points in $\omega(c)$ have only one preimage in $\omega(c))$; see [BKP].

Symbolic dynamics provides many examples of minimal noninvertible maps. Consider $A^{\mathbb{N}}$ endowed with the shift. It is easy to prove by a compactness argument that any transitive subshift (i.e., closed shift-invariant subset of $A^{\mathbb{N}}$ ) on which the shift acts bijectively is reduced to a periodic orbit. On the other hand by the Jewett-Krieger theorem there exist a variety of minimal subshifts, most of which do not consist of a periodic orbit; among them, 0-entropy as well as positive-entropy systems with various properties. Other examples are less abstract: one-sided Sturmian and Toeplitz systems are minimal subshifts, none of which is reduced to one periodic orbit (as a general reference see [LMa]).

None of the above mentioned examples of noninvertible minimal maps is on a manifold. On the interval there is no minimal map at all and it is well known that the circle admits a minimal homeomorphism but does not admit any noninvertible minimal map (see $[\mathrm{AK}]$ ). In Section 3 we prove that in the case of tori of dimension $n \geq 2$ the situation is different-in contrast to the case $n=1$ they admit minimal noninvertible maps. We prove that any minimal skew product homeomorphism of the torus having an asymptotic pair of points has an almost one-to-one factor which is a minimal noninvertible map of the torus (see Theorem 3.2). Then we apply the technique of factorization to the point distal homeomorphism of the torus of $\mathrm{M}$. Rees $[\mathrm{R}]$ to show the existence of a minimal point distal noninvertible map on the torus such that both an extension of it and a factor of it are minimal homeomorphisms of the torus (see Theorem 3.3). Finally, we show how to modify Rees' technique of extension of an irrational rotation of the torus from $[\mathrm{R}]$ in order to obtain a minimal noninvertible map of the torus (see Theorem 3.4 and, for all details, Appendix) $\left(^{1}\right)$.

$\left({ }^{1}\right)$ In the present paper we consider only compact spaces. Of course, the problem of the existence of minimal maps in noncompact spaces has also been studied. For instance, by [LCY], on the two-dimensional sphere minus a finite set there are no homeomorphisms whose full orbits are all dense, the nonexistence of minimal maps (i.e. maps whose forward orbits are all dense) being well known in much more general spaces [G], [HK]. 
To summarize: There are compact spaces that do not admit any minimal map, there are spaces that admit minimal homeomorphisms but do not admit any minimal noninvertible map and there are spaces that admit both minimal homeomorphisms and minimal noninvertible maps. The authors do not know whether there are spaces that admit minimal noninvertible maps but do not admit any minimal homeomorphism.

When we consider a continuous selfmap of a compact Hausdorff space, it turns out that there is a close connection between its minimality, invertibility and openness. In fact, in Section 2 we prove that every minimal map is feebly open and if it is even open then it is necessarily invertible and so, being a continuous bijective selfmap of a compact Hausdorff space, it is a homeomorphism (see Theorem 2.4).

Further, it is shown that if $f$ is minimal and $A \subseteq X$ then both $f(A)$ and $f^{-1}(A)$ share with $A$ those topological properties which describe how large a set is (see Theorem 2.5).

In topological dynamics almost one-to-one maps (extensions) attract a considerable attention (cf. $[\mathrm{FW}]$ ). In the present paper we prove that any minimal map in a compact metric space is almost one-to-one and, moreover, when restricted to a suitable invariant residual set it becomes a minimal homeomorphism (see Theorems 2.7 and 2.8).

Acknowledgements. The authors thank P. Arnoux, J. Auslander, F. Blanchard, H. Bruin, P. Le Calvez, P. Maličký, M. Rees and V. Sharko for useful discussions and remarks.

This paper was written in part during the first author's visit to Universidad de Chile and the first and the second authors' visit to the Institut de Mathématiques de Luminy. The first author was partially supported by the INTAS OPEN 97 grant 1843 and by the FONDECYT grant 7980040. The second author was partially supported by the Slovak grant agency, grant numbers $1 / 4015 / 97$ and $1 / 7424 / 2000$. The third author was partially supported by the FONDECYT grants 1980704 and 8990013. The support as well as the kind hospitality of Universidad de Chile and the Institut de Mathématiques de Luminy are very much appreciated.

2. Minimality, invertibility and openness. Let $X$ be a Hausdorff topological space and let $f: X \rightarrow X$ be continuous. The dynamical system $(X ; f)$ is called (topologically) minimal if there is no proper subset $M \subseteq X$ which is nonempty, closed and $f$-invariant (i.e., $f(M) \subseteq M$ ). In such a case we also say that the map $f$ itself is minimal. Note that the system $(X ; f)$ is minimal if and only if the (forward) orbit of every point from $X$ is dense in $X$. If $Y \subseteq X$ is nonempty, closed and $f$-invariant then 
$Y$ is called a minimal set of the system $(X ; f)$ if the system $\left(Y ;\left.f\right|_{Y}\right)$ is minimal $\left({ }^{2}\right)$.

Moreover, recall that the system $(X ; f)$ or the map $f$ itself is called (topologically) transitive if for every pair of nonempty open sets $U$ and $V$ in $X$, there is a positive integer $n$ such that $f^{n}(U) \cap V \neq \emptyset$. Clearly, minimality implies transitivity. Recall also that if $f$ is transitive then $f(X)$ is obviously dense in $X$ and if we additionally assume $X$ to be compact, then $f(X)$ is also compact. Hence $f(X)=X$. If $X$ has an isolated point and $f$ is transitive then $X$ is just a periodic orbit of $f$. If $X$ is a compact metric space without isolated points, then the above definition of transitivity is equivalent to the existence of a dense orbit (see [S]; for a survey of results on transitivity see, e.g., $[\mathrm{KS}])$.

Recall that a map is called open if it sends open sets to open sets and is called closed if it sends closed sets to closed sets. A map $f$ is called feebly open if for every nonempty open subset $U$ of $X$, there is a nonempty open subset $V$ of $X$ such that $V \subseteq f(U)$. It is easy to see that a map is feebly open if and only if the inverse image of every dense subset is dense. Note that the terminology is not unified-instead of feebly open some authors say semi-open, almost open or somewhat open. It seems that the idea of a feebly open map was first introduced in $[\mathrm{F}]$.

We will also use the notion of an irreducible map which is very important in general topology, mainly in the theory of absolutes (see e.g. [PW] or [Al]). A map $f: X \rightarrow Y$ is called irreducible if the only closed set $A \subseteq X$ for which $f(A)=Y$ is $A=X$. Note that if $f$ is irreducible then it is surjective.

Lemma 2.1. Let $X$ be a compact Hausdorff space and $f \in C(X)$. Then the following two conditions are equivalent and each of them is sufficient for $f$ not to be minimal:

(1) there is a closed set $A \neq X$ in $X$ with $f(A)=f(X)$,

(2) there is an open set $B \neq \emptyset$ in $X$ with $f(B) \subseteq f(X \backslash B)$. Consequently, if $f$ is minimal then it is irreducible.

Proof. From (1) we get (2) by taking $B=X \backslash A$ and from (2) we get (1) by taking $A=X \backslash B$. Suppose that (1) holds. If $f(X) \neq X$ then $f$ is obviously not minimal. So let $f(X)=X$. Denote $\left.f\right|_{A}$ by $g$ and consider the set $M:=\bigcap_{k=0}^{\infty} f^{-k}(A)=\bigcap_{k=0}^{\infty} g^{-k}(A)$. We have $X=g(A) \supseteq A$. Hence the set $M$, being the intersection of a nested sequence of nonempty compact sets,

$\left({ }^{2}\right)$ Thus in the present paper minimality of both a map and a homeomorphism means the density of all (forward) orbits. Some authors, when defining minimal homeomorphisms, only require the density of all full orbits (see, e.g., [W]) and speak of semiminimality when they have in mind the density of all (forward) orbits (see, e.g., [G]). In compact metric spaces these two notions are equivalent. 
is nonempty. But the $f$-trajectory of any point from $M$ does not intersect the nonempty open set $X \backslash A$ ( $M$ is even $f$-invariant and compact). Hence $f$ is not minimal.

Lemma 2.2. Let $X$ be a compact Hausdorff space and let $f \in C(X)$. Then the following are equivalent:

(1) $f$ is irreducible and open,

(2) $f$ is a homeomorphism.

Proof. Only $(1) \Rightarrow(2)$ needs a proof. Since a continuous bijection from a compact space to a Hausdorff space is a homeomorphism and $f$, being irreducible, is onto, it is sufficient to prove that $f$ is invertible. Suppose, on the contrary, that there are $a \neq b$ with $f(a)=f(b)=: c$. Take disjoint open neighbourhoods $U_{a}$ of $a$ and $U_{b}$ of $b$. Since $f$ is open, $f\left(U_{a}\right)$ is open and contains $c$. Since $f$ is continuous, there is an open neighbourhood $V_{b}$ of $b$ such that $V_{b} \subseteq U_{b}$ and $f\left(V_{b}\right) \subseteq f\left(U_{a}\right)$. Then $f\left(V_{b}\right) \subseteq f\left(X \backslash V_{b}\right)$. Hence, by Lemma $2.1(2) \Rightarrow(1), f$ is not irreducible.

Lemma 2.3. Let $X$ be a compact Hausdorff space and let $f \in C(X)$ be irreducible. Then $f$ is feebly open.

Proof. Since $f$ is continuous, for any $A \subseteq X$ we have $f(\bar{A}) \subseteq \overline{f(A)}$. This inclusion is in fact an equality, because $X$ is compact and thus $f$ is closed. Now let $D \subseteq X$ be dense in $X$. Since $f$ is onto, we have $f\left(f^{-1}(D)\right)=D$ and so

$$
f\left(\overline{f^{-1}(D)}\right)=\overline{f\left(f^{-1}(D)\right)}=\bar{D}=X .
$$

Since $f$ is irreducible, this implies that $\overline{f^{-1}(D)}=X$.

By Lemmas 2.1 and 2.3, if $f \in C(X)$ is minimal then it is irreducible and if $f$ is irreducible then it is feebly open. Easy examples on the interval show that the converse implications do not hold.

From Lemmas 2.1-2.3 we immediately get

TheOREM 2.4. Let $X$ be a compact Hausdorff space and $f \in C(X)$.

(1) If $f$ is minimal then it is feebly open.

(2) If $f$ is minimal and open then it is a homeomorphism.

Since we have seen that there are minimal maps which are not homeomorphisms, in (1) we cannot replace feebly open by open and in (2) we cannot replace open by feebly open.

Once we know that every minimal map is feebly open, we can ask whether this result can be extended to topologically transitive maps. The answer is negative - a topologically transitive map may not be feebly open [M] (nevertheless, every transitive interval map is trivially feebly open). 
To summarize: Any minimal map is of one of the following two kinds: either it is homeomorphism or it is noninvertible and nonopen (but feebly open). (It cannot be invertible and nonopen, since a continuous bijection from a compact space to a Hausdorff space is a homeomorphism, hence open. Further, it cannot be noninvertible and open by Theorem 2.4(2).)

Recall that a set is called residual if its complement is of first category. Further, a set $A$ has the Baire property (see [K1, p. 87] or [O, p. 19]) if it is the symmetric difference of an open set and a first category set, i.e., if it is of the form $A=(G \backslash B) \cup C$ where $G$ is open and $B$ and $C$ are first category sets or, equivalently, if it is of the form $A=(F \backslash P) \cup Q$ where $F$ is closed and $P$ and $Q$ are first category sets.

Theorem 2.4(1) together with Lemma 2.1 enable us to show that if $f$ is minimal and $A \subseteq X$ then both $f(A)$ and $f^{-1}(A)$ share some topological properties with the set $A$-namely the ones which describe how large a set is. For completeness, the next theorem contains also some known results.

TheOrem 2.5. Let $X$ be a compact Hausdorff space and let $f \in C(X)$ be a minimal map. Let $A \subseteq X$.

(1) If $A$ is dense then both $f(A)$ and $f^{-1}(A)$ are dense.

(2) If $A$ is nowhere dense then both $f(A)$ and $f^{-1}(A)$ are nowhere dense.

(3) If $A$ is a first category set then both $f(A)$ and $f^{-1}(A)$ are first category sets.

(4) If $A$ is a second category set then both $f(A)$ and $f^{-1}(A)$ are second category sets.

(5) If $A$ has the Baire property then both $f(A)$ and $f^{-1}(A)$ have the Baire property.

(6) If $A$ is residual then both $f(A)$ and $f^{-1}(A)$ are residual.

(7) If $A$ has nonempty interior then both $f(A)$ and $f^{-1}(A)$ have nonempty interiors.

(8) If $A$ is open then there is a positive integer $r$ with the property $\bigcup_{k=0}^{r} f^{-k}(A)=\bigcup_{k=0}^{r} f^{k}(A)=X$.

(9) If $A$ is open then there is an open set $B \subseteq X$ such that $B \subseteq f(A) \subseteq$ $\bar{B}$ (here $B$ may not be unique; the largest such set is always $B=\operatorname{int} f(A)$ ).

Proof. (1) is an easy consequence of the fact that $f$ is continuous, onto and feebly open.

(2) Let $A$ be nowhere dense. We are going to prove that both $f(A)$ and $f^{-1}(A)$ are nowhere dense. Since the closure of a nowhere dense set is nowhere dense, we may assume that $A$ is closed.

Since $A$ is nowhere dense, $X \backslash A$ is dense. By feeble openness of $f$, $f^{-1}(X \backslash A)=X \backslash f^{-1}(A)$ is dense. Since $f^{-1}(A)$ is closed, we get the nowhere density of $f^{-1}(A)$. 
Now suppose, on the contrary, that $f(A)$ is dense in a nonempty open set $G$. Since $f(A)$ is closed, we have $f(A) \supseteq G$. For the set $B=A \cap f^{-1}(G)$ we have $B \subseteq A$ and $f(B)=G$. Then $f^{-1}(G)$ is open and $f^{-1}(G) \supseteq B$. Since $B$ is nowhere dense, there is a nonempty open set $U$ such that $U \subseteq f^{-1}(G)$ and $U \cap B=\emptyset$. Then $f(X \backslash U) \supseteq f(B)=G \supseteq f(U)$. By Lemma 2.1, $f$ is not minimal, a contradiction.

(3) This follows from (2).

(4) From (3) we trivially deduce that $f(A)$ is of second category whenever $A$ is of second category. To prove that also $f^{-1}(A)$ is of second category, realize that due to the surjectivity of $f, f\left(f^{-1}(A)\right)=A$.

(5) Let $A$ have the Baire property, i.e., $A=(F \backslash P) \cup Q$ where $F$ is closed and $P, Q$ are of first category. Then $f^{-1}(A)=\left(f^{-1}(F) \backslash f^{-1}(P)\right) \cup f^{-1}(Q)$ and $f(A)=\left(f(F) \backslash P^{*}\right) \cup f(Q)$ where $P^{*} \subseteq f(P)$. Now use (3) and the fact that by continuity both $f(F)$ and $f^{-1}(F)$ are closed.

(6) follows from (3), (4) and the surjectivity of $f$.

(7) is a consequence of the continuity and the feeble openness of $f$.

(8) The existence of an $r$ with $\bigcup_{k=0}^{r} f^{-k}(A)=X$ is folklore (for any $x \in X$ there is $n(x)$ with $f^{n(x)}(x) \in A$ and so we have $f^{n(x)}\left(U_{x}\right) \subseteq A$ for some open neighbourhood $U_{x}$ of $x$; now use compactness to find a finite cover of $X$ by such neighbourhoods). Since $f$ is surjective we have $f\left(f^{-1}(B)\right)=B$ for any set $B$. Thus $X=f^{r}(X)=f^{r}\left(\bigcup_{k=0}^{r} f^{-k}(A)\right)=\bigcup_{k=0}^{r} f^{r-k}(A)=$ $\bigcup_{j=0}^{r} f^{j}(A)$.

(9) Take $B=\operatorname{int} f(A)$ and suppose on the contrary that for some $a \in A$, $f(a) \notin \bar{B}$. Take an open neighbourhood $V$ of $f(a)$ disjoint from $B$. By continuity, there is an open neighbourhood $U$ of $a$ such that $U \subseteq A$ and $f(U) \subseteq V$. By feeble openness of $f$, there is a nonempty open set $W$ with $W \subseteq f(U)$. Hence $W$ is a subset of $f(A)$ and is disjoint from $B=\operatorname{int} f(A)$, a contradiction.

REMARK. From the proof of Theorem 2.5 one can see that the statements (1)-(7) and (9) do not hold only for minimal maps but also for larger subclasses of $C(X)$. For instance, if we consider surjective maps then

- the $f$-part of (1) and the $f^{-1}$-part of (7) hold for all continuous maps,

- the $f^{-1}$-parts of (1), (2), (3), (5) and (6) as well as the $f$-parts of (4) and (7) hold for all feebly open maps,

- the $f$-parts of (2), (3), (5) and (6) as well as the $f^{-1}$-part of (4) hold for all irreducible maps,

- the statement (9) holds for all feebly open maps.

Note that for topologically transitive maps the theorem does not hold even on the interval (except the parts holding for all surjective feebly open maps; note that on the interval transitivity implies feeble openness). For instance, one can find a transitive feebly open map $f$ from a compact real 
interval $I$ into itself and a nowhere dense set $A \subset I$ such that $f(A)=I$ (take the Cantor set $A$ and the corresponding Cantor stairs map $I \rightarrow I$ and in each interval contiguous to $A$ replace the constant piece of the map by three linear pieces - increasing, decreasing, increasing; just take care of having sufficiently big "peaks" to ensure transitivity but not too big to ensure continuity).

For completeness let us remark that for continuous maps in compact topological spaces minimality (even surjectivity) implies another form of openness, so-called bicontinuity. A map $f: X \rightarrow X$ is called bicontinuous (or a factor map or an identification map) if it is onto and $f^{-1}(A)$ is open if and only if $A$ is open. In fact, if $X$ is compact and $f \in C(X)$ onto, then $f$, being a closed map, is bicontinuous (see [K1], p. 119).

Recall that a set is called regular closed or canonical closed if it is the closure of an open set (equivalently, if it is the closure of its interior). In the theory of absolutes it is important that if $X$ and $Y$ are topological spaces, $f: X \rightarrow Y$ is closed and irreducible and $A \subseteq X$ and $B \subseteq Y$ are regular closed sets, then $f(A)$ is regular closed and there is a unique regular closed set $B^{*}$ with $f\left(B^{*}\right)=B$ (see [Al], p. 345). By Lemma 2.1, if $X$ is compact Hausdorff and $f \in C(X)$ minimal then it also has the described properties.

Having proved Theorem 2.5 we are prepared to attack the problem of to what extent a minimal map may be noninvertible. We start with the following simple observation.

Lemma 2.6. Let $(X, \varrho)$ be a compact metric space and $f \in C(X)$ be onto. Then the map $\varphi: X \rightarrow[0, \operatorname{diam} X]$ defined by

$$
\varphi(x)=\operatorname{diam} f^{-1}(x)
$$

is upper semicontinuous.

Proof. Fix a point $x_{0} \in X$. To prove that $\limsup _{x \rightarrow x_{0}} \varphi(x) \leq \varphi\left(x_{0}\right)$ assume that $\varphi\left(z_{n}\right) \rightarrow d$ for some sequence $z_{n} \rightarrow x_{0}$. We need to show that $d \leq \varphi\left(x_{0}\right)$. For any $n$ take points $p_{n}$ and $q_{n}$ from the compact set $f^{-1}\left(z_{n}\right)$ such that $\varrho\left(p_{n}, q_{n}\right)=\varphi\left(z_{n}\right)$. Since we are in a compact metric space, there are subsequences $p_{k_{n}}$ and $q_{k_{n}}$ converging to some points $p$ and $q$. Then $\varrho(p, q)=\lim _{n \rightarrow \infty} \varrho\left(p_{k_{n}}, q_{k_{n}}\right)=\lim _{n \rightarrow \infty} \varphi\left(z_{k_{n}}\right)=d$. Further, $f(p)=$ $\lim _{n \rightarrow \infty} f\left(p_{k_{n}}\right)=\lim _{n \rightarrow \infty} z_{k_{n}}=x_{0}$ and similarly $f(q)=x_{0}$. Hence $p, q \in$ $f^{-1}\left(x_{0}\right)$ and so $d \leq \varphi\left(x_{0}\right)$.

Recall that a map $f: X \rightarrow X$ is called almost one-to-one if for every $x$ in a $G_{\delta^{-}}$-dense subset of $X, \operatorname{card}\left(f^{-1}(x)\right)=1$. Equivalently, in this definition instead of " $G_{\delta^{-}}$-dense" we can use "residual", because any $G_{\delta^{-}}$-dense set is residual and any residual set (in a compact metric space) contains a $G_{\delta}$-dense set. Thus, a map is called almost one-to-one if generically the preimage of a point is a singleton. 
The following theorem sheds some light on the problem of to what extent a minimal map may be noninvertible. It shows that for minimal maps the set of points which have more than one preimage is of first category. In fact, we have

Theorem 2.7. Let $(X, \varrho)$ be a compact metric space and $f \in C(X)$ be minimal. Then the set $A=\left\{x \in X: \operatorname{card} f^{-1}(x)=1\right\}$ is a $G_{\delta}$-dense set in $X$. Hence, $f$ is almost one-to-one.

Proof. Consider the map $\varphi$ from Lemma 2.6. Notice that $A=\bigcap_{k=1}^{\infty} A_{k}$, where $A_{k}=\varphi^{-1}([0,1 / k))$. Since $\varphi$ is upper semicontinuous, $A_{k}$ is open in $X$ for every $k$. By the Baire theorem the intersection of countably many open dense sets in $X$ is $G_{\delta}$-dense, therefore to finish the proof it is sufficient to prove that for every $k$ the set $A_{k}$ is dense. Suppose, on the contrary, that for some $k$, the (closed) set $D_{k}=X \backslash A_{k}=\left\{x \in X: \operatorname{diam} f^{-1}(x) \geq 1 / k\right\}$ has nonempty interior. Then also $f^{-1}\left(D_{k}\right)$ has nonempty interior. Take a nonempty open set $B \subseteq f^{-1}\left(D_{k}\right)$ such that $\operatorname{diam} B<1 / k$. The set $B$ is covered by the preimages of some points from $D_{k}$. Each of these preimages has diameter $\geq 1 / k$ and so cannot be placed entirely in $B$. Hence $f(B) \subseteq$ $f(X \backslash B)$. By Lemma 2.1, $f$ is not minimal, a contradiction.

The above theorem enables us to show that a "substantial" part of a minimal map is a minimal homeomorphism.

Theorem 2.8. Let $(X, \varrho)$ be a compact metric space and $f \in C(X)$ be minimal. Then there exists a residual set $Y \subseteq X$ such that $f(Y)=Y$ and $\left.f\right|_{Y}$ is a minimal homeomorphism. Moreover, $\left(\left.f\right|_{Y}\right)^{-1}$ is also a minimal homeomorphism and while $\left.f\right|_{Y}$ is uniformly continuous, $\left(\left.f\right|_{Y}\right)^{-1}$ is uniformly continuous only in the case when $f$ is a homeomorphism (then one can take $Y=X)$.

Proof. By Theorem 2.7, the set $A=\left\{x \in X: \operatorname{card} f^{-1}(x)=1\right\}$ is $G_{\delta}$-dense and so $D=\left\{x \in X: \operatorname{card} f^{-1}(x)>1\right\}$ is of first category. Using Theorem 2.5(6) and the fact that the intersection of countably many residual sets is again residual we see that also the set $Y=\bigcap_{n=-\infty}^{\infty} f^{n}(A)$ is residual. (One can also notice that $Y=X \backslash \bigcup_{n=-\infty}^{\infty} f^{n}(D)$.)

It is not difficult to see that $y_{0} \in Y$ if and only if the full orbit of $y_{0}$, i.e. the set $\left\{x \in X: \exists i, j \in \mathbb{N}\right.$ with $\left.f^{i}(x)=f^{j}\left(y_{0}\right)\right\}$, is of the form $\left\{\ldots, y_{-2}, y_{-1}, y_{0}, y_{1}, y_{2}, \ldots\right\}$ where $f\left(y_{n}\right)=y_{n+1}$ for every integer $n$. From this we immediately see that $f(Y)=Y$ and $\left.f\right|_{Y}$ is a bijection. Trivially, $\left.f\right|_{Y}$ is continuous. We claim that also $\left(\left.f\right|_{Y}\right)^{-1}$ is continuous. To see it, suppose that $\left(\left.f\right|_{Y}\right)^{-1}$ is not continuous at a point $y_{0} \in Y$. Then we can find a sequence $z_{i}$ in $Y$ such that $z_{i} \rightarrow y_{0}$ and $f^{-1}\left(z_{i}\right) \not f^{-1}\left(y_{0}\right)$. Considering now $f^{-1}\left(z_{i}\right)$ in the compact metric space $X$, we can assume that $f^{-1}\left(z_{i}\right) \rightarrow$ $z \neq f^{-1}\left(y_{0}\right)$. Since both $f(z)$ and $f\left(f^{-1}\left(y_{0}\right)\right)$ equal $y_{0}$, we deduce that 
$y_{0} \in D$ and so $y_{0} \notin Y$, a contradiction. Thus $\left.f\right|_{Y}$ is a homeomorphism. The minimality of $\left.f\right|_{Y}$ is obvious, since it is a restriction of a minimal map.

Now consider $\left(\left.f\right|_{Y}\right)^{-1}$. Obviously, it is a homeomorphism $Y \rightarrow Y$. We are going to prove its minimality. Suppose, on the contrary, that for some point $y_{0} \in Y$ its $\left(\left.f\right|_{Y}\right)^{-1}$-trajectory is not dense in $Y$. Denote by $\omega$ its $\omega$-limit set (in $X$ ), i.e. the set of all points in $X$ which are limit points of this trajectory. Trivially, $\omega$ is nonempty and closed in $X$ and by the above, $\omega \neq X$. Taking $a \in \omega$ and using the continuity of $f$ one can find $b \in \omega$ with $f(a)=b$. Hence $f(\omega) \subseteq \omega$, a contradiction with the minimality of $f$.

Finally, let us turn to uniform continuity. Since $f$ is uniformly continuous, so is $\left.f\right|_{Y}$. If $f$ is a homeomorphism (i.e., $Y=X$ ) then so is $f^{-1}$ and by compactness we get the uniform continuity of $\left.f^{-1}\right|_{Y}=\left(\left.f\right|_{Y}\right)^{-1}$. Conversely, let $\left(\left.f\right|_{Y}\right)^{-1}$ be uniformly continuous. Then it has a continuous extension $g \in C(X)$ and obviously $\left.g \circ f\right|_{Y}=\left.f \circ g\right|_{Y}=\left.\mathrm{id}\right|_{Y}$. Since $Y$ is dense in $X$, this implies $g \circ f=f \circ g=$ id on $X$. Hence $f$ is a homeomorphism.

REMARK. From the proof of Theorem 2.7 one can see that it holds under weaker assumptions - it is sufficient to assume that $f \in C(X)$ is irreducible. (One can compare this result with that of $[\mathrm{MW}]$ where, though under weaker assumptions, only the density of $A$ is obtained.)

Similarly, if in Theorem 2.8 we only assume irreducibility of the map $f \in C(X)$ then the statement remains valid except for the minimality of $\left.f\right|_{Y}$ and $\left(\left.f\right|_{Y}\right)^{-1}$. One can only claim that these maps, being bijective, are irreducible.

Finally we remark that if $f$ in Theorem 2.8 is not a homeomorphism, i.e., we cannot put $Y=X$, then the set $Y$ has necessarily empty interior in $X$ (use for instance Theorem 2.5(8) and the fact that $f(Y)=Y$ ).

J. Auslander brought our attention to the fact that there is a valid converse to Theorem 2.8. In fact, we have

TheOREM 2.9. Let $(Y, \tau)$ be a metric space and $h: Y \rightarrow Y$ be a minimal homeomorphism such that

(a) $h$ is uniformly continuous,

(b) $h^{-1}$ is not uniformly continuous and

(c) for any $\varepsilon>0$ there is a nonnegative $N$ such that for every $y \in Y$, the set $\left\{y, h(y), \ldots, h^{N}(y)\right\}$ is $\varepsilon$-dense in $Y$.

Then $(Y, \tau)$ is not complete, its completion $(X, \varrho)$ is compact (hence a compactification of $(Y, \tau))$, and there is a minimal noninvertible map $f$ on $X$ which extends $h$.

Proof. Suppose that $(Y, \tau)$ is complete. By (c) it is also totally bounded and thus compact. But then $h^{-1}$ is uniformly continuous on $Y$, a contradiction. 
Let $(X, \varrho)$ be the completion of $(Y, \tau)$. Since $Y$ is totally bounded, so is $X$. Hence $X$ is compact.

The map $h: Y \rightarrow X$ is uniformly continuous and $Y$ is dense in the compact (hence complete) metric space $X$. Thus there is a (uniformly) continuous extension $f: X \rightarrow X$ of $h$. Obviously, $f$ is onto because $f(X) \supseteq$ $h(Y)=Y, Y$ is dense in $X$ and $f(X)$ is compact. The map $f$ cannot be invertible on $X$ since otherwise $h^{-1}$ would be uniformly continuous on $Y$.

Finally, from (c) we conclude, using uniform continuity of $f$, that for any $\varepsilon>0$ there is a nonnegative $N$ such that for every $x \in X$, the set $\left\{x, h(x), \ldots, h^{N}(x)\right\}$ is $\varepsilon$-dense in $X$. The minimality of $f$ trivially follows.

Remark. Notice that in Theorem 2.9, up to isometry there is no other compactification $(\widetilde{X}, \widetilde{\varrho})$ of $(Y, \tau)$ for which the metric $\widetilde{\varrho}$ induces the given metric $\tau$ on $Y$. In fact, any such compactification is automatically the completion of $(Y, \tau)$.

3. Noninvertible minimal maps on the torus. We are going to prove that the torus admits minimal noninvertible maps. Two kinds of examples of such maps will be given - they will be obtained either as a factor or as an extension of an appropriate minimal homeomorphism of the torus.

We start with some preliminaries.

Above we worked with almost one-to-one maps. Similar terminology is also used for extensions and factors. Let $f: X \rightarrow X, g: Y \rightarrow Y$ and $\varphi: X \rightarrow Y$ be continuous, with $\varphi$ surjective. Let $\varphi \circ f=g \circ \varphi$, i.e., $\varphi$ is a semiconjugacy, $f$ is an extension of $g$ and $g$ is a factor of $f$. This extension of $g$ (factor of $f$ ) is called almost one-to-one if the semiconjugacy $\varphi$ is an almost one-to-one map, i.e., if for every $y$ in a residual subset of $Y, \operatorname{card} \varphi^{-1}(y)=1$.

Let $(X, \varrho)$ be a metric space and $f: X \rightarrow X$ be continuous. Then two different points $x, y \in X$ are called distal if $\liminf _{n \rightarrow \infty} \varrho\left(f^{n} x, f^{n} y\right)>0$, proximal if $\liminf _{n \rightarrow \infty} \varrho\left(f^{n} x, f^{n} y\right)=0$ and asymptotic if $\lim _{n \rightarrow \infty} \varrho\left(f^{n} x, f^{n} y\right)=$ 0 . The map $f$ is called distal if any two different points from $X$ are distal, and is called point distal if there is a point $x_{0} \in X$ such that for every $y \in X$, $y \neq x_{0}$, the points $x_{0}, y$ are distal. If $f$ is distal then it is point distal but, in general, not conversely. If a misunderstanding can arise, we say $f$-distal instead of distal and similarly for proximality and asymptoticity.

A decomposition $\mathcal{D}$ of a topological space $X$ is upper semicontinuous (u.s.c.) if for each element $E$ in $\mathcal{D}$ and each open set $U$ containing $E$ there is an open set $V$ such that $E \subset V \subset U$ and $V$ is the union of members of $\mathcal{D}$.

Further we need to recall the Roberts-Steenrod theorem from [RS]. Let $M$ be a compact connected 2-dimensional manifold without boundary (it need not be orientable) and $G$ be an u.s.c. collection of continua filling $M$. Let $R(g)$ denote the mod 2 one-dimensional Betti number of the set $g$. 
Theorem 1 of [RS] says that if $G$ contains at least two elements and $R(g)=0$ for each $g \in G$, then the quotient space $M / G$ is homeomorphic to $M$. (One can easily see that the topology used by Roberts and Steenrod is really the quotient topology in the contemporary terminology.)

A continuous map $S$ from the 2-torus $\mathbb{T}^{2}$ into itself is called a skew prod$u c t$ if it is of the form $S(x, y)=(f(x), g(x, y))$. Obviously, if $S$ is minimal then so is the circle map $f$. But then $f$ is topologically conjugate to an irrational rotation $x \mapsto x+\alpha$ (see $[\mathrm{AK}]$ ). A set of the form $\left\{x_{0}\right\} \times I \subseteq \mathbb{T}^{2}$ where $I$ is an interval on the circle is said to be a vertical interval on the torus.

The following lemma admits generalizations but is sufficient for our purposes.

Lemma 3.1. Let $\Phi$ be a minimal skew product continuous selfmap of the 2 -torus $\mathbb{T}^{2}$. Assume that one of the following holds:

(a) There are (possibly degenerate) compact vertical intervals $J_{n}, n=$ $0,1,2, \ldots$, such that $\Phi\left(J_{n}\right)=J_{n+1}$ for every $n$, and $\operatorname{diam} J_{n} \rightarrow 0$ as $n \rightarrow$ $+\infty$.

(b) There are (possibly degenerate) compact vertical intervals $J_{n}, n=$ $0,-1,-2, \ldots$, such that $J_{0}$ is a singleton and $\Phi\left(J_{n}\right)=J_{n+1}$ for $n=-1$, $-2, \ldots$, and $\operatorname{diam} J_{n} \rightarrow 0$ as $n \rightarrow-\infty$.

Let $\mathcal{D}$ be the decomposition of $\mathbb{T}^{2}$ whose elements are the intervals $J_{n}$ and the individual points from the rest of the torus. Then the quotient space $\mathbb{T}^{2} / \mathcal{D}$ is homeomorphic to $\mathbb{T}^{2}$ and there exists a natural almost one-to-one factor $\Psi: \mathbb{T}^{2} / \mathcal{D} \rightarrow \mathbb{T}^{2} / \mathcal{D}$ of the map $\Phi$ (the corresponding semiconjugacy is the natural projection $\left.p: \mathbb{T}^{2} \rightarrow \mathbb{T}^{2} / \mathcal{D}\right)$. Moreover, $\Psi$ is a continuous minimal map.

Proof. According to the discussion preceding the lemma, the intervals are pairwise disjoint and so the decomposition is well defined. In either of the cases (a) and (b), $\Phi$ maps an element of the decomposition $\mathcal{D}$ into an element of $\mathcal{D}$. Hence there is a unique map $\Psi: \mathbb{T}^{2} / \mathcal{D} \rightarrow \mathbb{T}^{2} / \mathcal{D}$ with $\Psi \circ p=p \circ \Phi$. We prove that $\Psi$ is continuous. Since the map $h=p \circ \Phi$ : $\mathbb{T}^{2} \rightarrow \mathbb{T}^{2} / \mathcal{D}$ is continuous and $h=\Psi \circ p$, for each open set $U \subseteq \mathbb{T}^{2} / \mathcal{D}$, $h^{-1}(U)=p^{-1}\left(\Psi^{-1}(U)\right)$ is open in $\mathbb{T}^{2}$. Therefore, since $p$ is a quotient map, the set $\Psi^{-1}(U)$ is open in $\mathbb{T}^{2} / \mathcal{D}$. Thus $\Psi$ is continuous and, being a factor of a minimal map, it is also minimal. Obviously, it is an almost one-to-one factor of $\Phi$.

Thus to finish the proof we need to show that $\mathbb{T}^{2} / \mathcal{D}$ is homeomorphic to $\mathbb{T}^{2}$. To this end we apply the above mentioned Roberts-Steenrod theorem. Since the mod 2 one-dimensional Betti number of every element of $\mathcal{D}$ is zero, the only thing which remains to be proved is that the partition $\mathcal{D}$ is u.s.c. 
First realize that in either of the cases (a) and (b) the decomposition $\mathcal{D}$ consists of individual points and of a sequence of closed (possibly degenerate) one-dimensional intervals $K_{n}, n=0,1,2, \ldots$, with diam $K_{n} \rightarrow 0$ as $n \rightarrow$ $\infty$. Take any element $E$ of $\mathcal{D}$ and any open set $U$ containing $E$. Since $E$ is compact, without loss of generality we may assume that $U$ is a $\delta$ neighbourhood of $E$ for some $\delta>0$. Now let $Y$ be the union of all elements of $\mathcal{D}$ which intersect the boundary of $U$. Obviously, $Y \supseteq$ bd $U$. To finish the proof that $\mathcal{D}$ is u.s.c. it is obviously sufficient to show that $Y$ is closed. To this end let $\left(y_{n}\right)_{n=1}^{\infty}$ be a converging sequence of points from $Y$ and $y$ be its limit. Denote by $D_{n}$ the element of $\mathcal{D}$ containing $y_{n}$. If for infinitely many $n$ 's the point $y_{n}$ is contained in the same element $D_{r}$ then $y \in D_{r} \subseteq Y$ and we are done. So let there be a subsequence $\left(D_{n_{k}}\right)_{k=1}^{\infty}$ of mutually different elements. From the fact that $\operatorname{diam} K_{n} \rightarrow 0$ it follows that also the diameters of $D_{n_{k}}$ tend to zero, whence $\lim _{k \rightarrow \infty} y_{n_{k}}=y \in \operatorname{bd} U$ and so again $y \in Y$.

Now we are ready to prove that the torus admits noninvertible minimal maps. Since there are minimal skew product homeomorphisms of the torus having asymptotic pairs of points (an example of such a homeomorphism is in $[R])$, it is sufficient to prove the following

TheORem 3.2. Any minimal skew product homeomorphism of the 2torus $\mathbb{T}^{2}$ having an asymptotic pair of points has an almost one-to-one factor which is a noninvertible minimal map of $\mathbb{T}^{2}$.

Proof. Let $S(x, y)=(f(x), g(x, y))$ be a minimal skew product homeomorphism of $\mathbb{T}^{2}$. Assume that $S$ has an asymptotic pair of points $\left\{z_{1}, z_{2}\right\}$. Of course, these points lie in one fibre, i.e., they are of the form $z_{1}=\left(x, y_{1}\right)$, $z_{2}=\left(x, y_{2}\right)$.

Since $S$ is a homeomorphism of the above form, the $S$-image of a vertical interval is again a vertical interval whose endpoints are the $S$-images of the endpoints of the original interval. The points $z_{1}, z_{2}$ are the endpoints of two vertical compact intervals. Since these points are asymptotic and $S$ is uniformly continuous, one of the above mentioned two vertical intervals, denote it by $I_{0}$, is such that for $I_{n}:=S^{n}\left(I_{0}\right)$ we have diam $I_{n} \rightarrow 0$ as $n \rightarrow+\infty$.

Let $\mathcal{D}$ be the decomposition of $\mathbb{T}^{2}$ whose elements are the (pairwise disjoint) compact intervals $I_{n}, n \geq 0$, and the individual points from $\mathbb{T}^{2} \backslash$ $\bigcup_{n=0}^{\infty} I_{n}$. Consider the quotient space $\mathbb{T}^{2} / \mathcal{D}$.

By identifying each of the intervals $I_{n}, n \geq 0$, to a single point we get, by Lemma 3.1, an almost one-to-one factor $F: \mathbb{T}^{2} / \mathcal{D} \rightarrow \mathbb{T}^{2} / \mathcal{D}$ of the map $\Phi$. The map $F$ is continuous, minimal and noninvertible at the point $p\left(I_{0}\right)$.

To finish the proof realize that, by Lemma $3.1, \mathbb{T}^{2} / \mathcal{D}$ is homeomorphic to $\mathbb{T}^{2}$. 
The technique from Lemma 3.1 applied to the map from $[R]$ can be used to prove the following

THEOREM 3.3. There is a minimal point distal noninvertible map of the 2-torus $\mathbb{T}^{2}$ such that:

(1) an extension of it is a minimal point distal skew product homeomorphism of $\mathbb{T}^{2}$ and

(2) a factor of it is a minimal distal homeomorphism of $\mathbb{T}^{2}$.

Proof. The three minimal maps will be denoted by $S, F$ and $H, F$ being noninvertible and homeomorphisms $S$ and $H$ being an extension and a factor of $F$, respectively.

Let $S$ be the minimal point distal but not distal skew product homeomorphism from $[R]$. It is obtained as an extension of an irrational rotation of $\mathbb{T}^{2}$. We will use the following properties of $S$. There is a vertical nondegenerate compact interval $I_{0}$ such that for $I_{n}:=S^{n}\left(I_{0}\right)$ we have diam $I_{n} \rightarrow 0$ as $n \rightarrow \infty$ as well as $n \rightarrow-\infty$. There are no $S$-proximal pairs which are not $S$-asymptotic. Two different points $x, y \in \mathbb{T}^{2}$ are $S$-asymptotic if $x, y \in I_{n}$ for some integer $n$ and are $S$-distal otherwise.

The identification of each of the intervals $I_{n}, n \geq 0$, to a single point leads, in view of Lemma 3.1, to a minimal continuous selfmap $F$ of $\mathbb{T}^{2}$, a factor of $S$. Obviously, $F$ is noninvertible (at the point which corresponds to the interval $I_{0}$ ).

Quite analogously, the further identification of the intervals $I_{n}, n<0$, to singletons leads to a minimal homeomorphism $H$ of $\mathbb{T}^{2}$, a factor of $F$.

Thus $H: \mathbb{T}^{2} \rightarrow \mathbb{T}^{2}$ is a factor of $S$ which is topologically conjugate to another factor of $S$, the map $\mathbb{T}^{2} / \mathcal{D} \rightarrow \mathbb{T}^{2} / \mathcal{D}$ obtained from $S$ by collapsing all the elements of $\mathcal{D}$ to singletons, where $\mathcal{D}$ consists of the intervals $\left(I_{n}\right)_{n=-\infty}^{\infty}$ and the individual points from the rest of the torus (we use the fact that, by Lemma 3.1, $\mathbb{T}^{2}$ and $\mathbb{T}^{2} / \mathcal{D}$ are homeomorphic). Hence there is a continuous surjective map $\pi: \mathbb{T}^{2} \rightarrow \mathbb{T}^{2}$ with $\pi \circ S=H \circ \pi$ (the level sets of $\pi$ are the elements of $\mathcal{D})$. We are going to show that $H$ is distal.

Suppose, on the contrary, that two different points $x, y \in \mathbb{T}^{2}$ are $H$ proximal. Since $x \neq y, A=\pi^{-1}(x)$ and $B=\pi^{-1}(y)$ are different elements of $\mathcal{D}$. Pick $a \in A, b \in B$. Then $a, b$ are $S$-distal. Thus for some $\delta>0$ we have $\varrho\left(S^{n} a, S^{n} b\right) \geq \delta, n=0,1,2, \ldots$, and $\liminf _{n \rightarrow \infty} \varrho\left(H^{n} x, H^{n} y\right)=0$. By a compactness argument there is a sequence $n_{k} \rightarrow \infty$ and points $c, d$ and $z$ such that

$$
H^{n_{k}} x \rightarrow z, \quad H^{n_{k}} y \rightarrow z, \quad S^{n_{k}} a \rightarrow c, \quad S^{n_{k}} b \rightarrow d .
$$

The semiconjugacy gives $\pi(c)=z$ as well as $\pi(d)=z$. Thus $c, d$ lie in the same interval $I_{n}$. Therefore they are $S$-asymptotic. Hence, for some $m$, $\varrho\left(S^{m} a, S^{m} b\right)<\delta$, a contradiction. 
To show the existence of noninvertible minimal maps on the torus we were using the technique of factorization (see Theorems 3.2 and 3.3). Nevertheless, taking an extension of a minimal homeomorphism can also yield a noninvertible minimal map. In fact, we are going to show how to modify the construction of $\mathrm{M}$. Rees $[\mathrm{R}]$ to obtain a noninvertible minimal map of the torus. (In the first part of the proof of Theorem 3.3 we showed that the map $S$ from $[\mathrm{R}]$ has a factor $F$ which is a noninvertible minimal map of the torus. Now we want to find such a map without using factorization.)

Theorem 3.4. Any irrational rotation of the 2-torus $\mathbb{T}^{2}$ has an almost one-to-one extension which is a noninvertible minimal map of $\mathbb{T}^{2}$.

Idea of the proof. Extend an irrational rotation of the torus analogously to $[\mathrm{R}]$ but in the doubly infinite sequence of squares ..., $A_{3}, A_{1}, A_{0}$, $A_{2}, A_{4}, \ldots$ let $A_{2 n}, n=1,2, \ldots$, be degenerate (i.e., points). (In [R] all the squares are nondegenerate.)

Just pointing out the differences between the proof of Theorem 3.4 and that from $[R]$ may not seem sufficient. In fact, the authors of the present paper find the paper $[R]$ very difficult to read - in particular, there are many misprints and gaps there. On the other hand the map and the construction from $[R]$ are very interesting and exhibit nice properties. It seems that in spite of that they are often overlooked and are not widely known. All things considered, we have decided to present a detailed proof of Theorem 3.4 as an appendix.

4. Appendix - detailed proof of Theorem 3.4. Let $T:(x, y) \mapsto$ $(x+\alpha, y+\beta)$ be a minimal homeomorphism of the 2 -torus $\mathbb{T}^{2}=\mathbb{R}^{2} / \mathbb{Z}^{2}$, where $1, \alpha, \beta \in \mathbb{R}$ are rationally independent and $+: \mathbb{R} / \mathbb{Z} \times \mathbb{R} \rightarrow \mathbb{R} / \mathbb{Z}$ is defined in the obvious way. The circle $\mathbb{R} / \mathbb{Z}$ will be denoted by $K$. Let $\pi: \mathbb{T}^{2} \rightarrow K$ be the projection $(x, y) \mapsto x$. If $z=(x, y)$ is a point from the torus we will denote the set $\{x\} \times K=\{\pi(z)\} \times K$ by $K_{x}$ and sometimes also by $K_{z}$ (we hope that no confusion can arise since it will always be clear whether the subscript is a point from the torus or only its first coordinate).

Let $z_{0}=\left(x_{0}, y_{0}\right) \in \mathbb{T}^{2}$ and for $n \geq 0$ let $z_{2 n}=\left(x_{2 n}, y_{2 n}\right)=T^{n}\left(x_{0}, y_{0}\right)$ and $z_{2 n+1}=\left(x_{2 n+1}, y_{2 n+1}\right)=T^{-n-1}\left(x_{0}, y_{0}\right)$. So, the trajectory of the point $z_{0}$ is $\ldots, z_{3}, z_{1}, z_{0}, z_{2}, z_{4}, \ldots$ Set $X=\mathbb{T}^{2} \backslash \bigcup_{n=-\infty}^{\infty} K_{z_{n}}$.

For $x, y \in K$, if $x=\mathbb{Z}+a, y=\mathbb{Z}+b(a, b \in \mathbb{R})$, then let $|x-y|=$ $\inf _{p \in \mathbb{Z}}|a-b+p|$. If $z_{1}=\left(x_{1}, y_{1}\right) \in \mathbb{T}^{2}, z_{2}=\left(x_{2}, y_{2}\right) \in \mathbb{T}^{2}$, let $\left|z_{1}-z_{2}\right|=$ $\max \left\{\left|x_{1}-x_{2}\right|,\left|y_{1}-y_{2}\right|\right\}$. If $f$ and $g$ are continuous selfmaps of the torus, we define their distance by $\|f-g\|=\max _{z \in \mathbb{T}^{2}}|f(z)-g(z)|$. The identity map will be denoted by id. If $A$ is a square, $|A|$ will denote the length of its side. Sometimes we will use the notation $\bar{r}, s$ for the set of integers $\{r, r+1, \ldots, s\}$. The symbol $\mathbb{N}$ will denote the set of nonnegative integers. 
(A) $-(\mathbf{H})$ Definition of a sequence of squares $\left(A_{n}\right)_{n=0}^{\infty}$ and sequences of maps $\left(\Lambda_{n}\right)_{n=0}^{\infty}$ and $\left(\Phi_{n}\right)_{n=0}^{\infty}$. The square $A_{n}$ will have centre $z_{n}$. The lengths of the sides of the squares will be chosen to satisfy several conditions (see below).

(A) Define a family $h_{t}, t \in[-1,1]$, of continuous functions $[-1,1] \rightarrow$ $[-1,1]$ as follows. Let $h_{0}$ be the piecewise linear map consisting of three pieces, whose graph contains the points $[-1,-1],[-1 / 2,0],[1 / 2,0],[1,1]$. For $t \neq 0$ let $h_{t}$ be the piecewise linear map consisting of five pieces (of one piece if $t= \pm 1$ ) whose graph contains the points $[-1,-1],\left[h_{0}^{-1}(t), t\right],[-1 / 2,-|t| / 2]$, $[1 / 2,|t| / 2],\left[h_{0}^{-1}(-t),-t\right],[1,1]$. Note that $h_{-1}=h_{1}=\mathrm{id}, h_{-t}=h_{t}$ and only $h_{0}$ is not a homeomorphism, $h_{0}^{-1}(0)=[-1 / 2,1 / 2]$.

(B) Let $A \subseteq \mathbb{T}^{2}$ be a square with $|A|=2 \delta>0$ and with centre $z_{A}$. Define $\Lambda_{A}=$ id outside $A$, and

$$
\Lambda_{A}\left(z_{A}+(t, s)\right)=z_{A}+\left(t, h_{t} \circ h_{\delta}^{-1}(s)\right)
$$

for all $(t, s) \in[-\delta, \delta]^{2}$. It is easy to check that $\Lambda_{A}$ is the identity on the boundary of $A$, hence continuous. Notice also that $\Lambda_{A}$ does not change the first coordinate of points of the torus. Since $h_{t}^{-1}(y)$ is a singleton except for the case when $t=0$ and $y=0$, the inverse map $\Lambda_{A}^{-1}$ exists everywhere except at the point $z_{A}$. Moreover, one can see that $\Lambda_{A}^{-1}$ is continuous on $\mathbb{T}^{2} \backslash\left\{z_{A}\right\}$.

Further, if the square $A$ is degenerate to a point, $\Lambda_{A}$ will denote the identity on the torus. Then $\Lambda_{A}^{-1}=\mathrm{id}$ on the whole torus.

Put $I_{z_{A}}=\Lambda_{A}^{-1}\left(z_{A}\right)$.

(C) For any finite system of squares $A_{0}, \ldots, A_{n}$ we define $\Phi_{n}=\Lambda_{n} \circ$ $\ldots \circ \Lambda_{1} \circ \Lambda_{0}$, where $\Lambda_{i}$ stands for $\Lambda_{A_{i}}$.

(D) Let $\left|A_{2 n}\right|=0$ for $n=1,2, \ldots$, i.e., $A_{2 n}=\left\{z_{2 n}\right\}$ is a degenerate square. (This is the only formal difference between our construction and that from $[\mathrm{R}]$ where $\left|A_{n}\right|$ is never zero.)

(E) For any $n$ let $\left|A_{n}\right|<\frac{1}{2} \min \left\{\left|x_{m}-x_{p}\right|: m \neq p, m, p \leq n+5\right\}$. Then, for any $n, A_{n}$ does not intersect $K_{x_{i}}, i \in \overline{0, n-1} \cup \overline{n+1, n+5}$, and $A_{n} \cap A_{m}=\emptyset$ whenever $|m-n| \leq 5$. Note that for such $n, m$ we therefore have $\Lambda_{n} \circ \Lambda_{m}=\Lambda_{m} \circ \Lambda_{n}$, which will be used later. Note also that even the sets $\pi\left(\bar{A}_{i}\right) \times K, i=\overline{k, k+5}$, are pairwise disjoint, where $\bar{A}_{i}$ is the square with centre $z_{A}$ and with side length equal to the largest of the side lengths of the squares $A_{k}, \ldots, A_{k+5}$.

(F) Let $\left|A_{n}\right|<1 / 2^{n}, n=0,1,2, \ldots$

(G) Further, let $\left|A_{n}\right|$ be so small that $\left|\Phi_{r-1}^{-1}\left(\left(a_{r}+1\right) A_{r}\right)\right|<1 / r$ where $\left(a_{r}\right)_{r=1}^{\infty}$ is a fixed sequence of integers with $a_{r}>2$ and $\prod_{r=1}^{\infty}\left(1-2 / a_{r}\right)>0$, 
and $\left(a_{r}+1\right) A_{r}$ denotes the square with the same centre as $A_{r}$, and sides $a_{r}+1$ times as long.

(H) To formulate the next condition for the sides of the squares $A_{n}$ we need some notation. First, fix a sequence $\left(A_{n}^{*}\right)_{n=0}^{\infty}$ of squares satisfying the conditions $(\mathrm{A})-(\mathrm{G})$. For $s \geq 0$ let $M_{s}$ be a closed subset of the torus which does not contain the points $z_{i}, i=\overline{0, s}$, contains the squares $A_{j}^{*}, j=$ $\overline{s+1, s+5}$, (this is possible by $(\mathrm{E})$ ) and $T\left(A_{s+3}^{*}\right) \cup T^{-1}\left(A_{s+3}^{*}\right) \subseteq M_{s}$ (this is also possible by $(\mathrm{E}))$.

The map $\Phi_{s}^{-1}$ is defined and is continuous on $\mathbb{T}^{2} \backslash\left\{z_{0}, \ldots, z_{s}\right\}$. The map $\Phi_{s}^{-1}$ is not uniformly continuous but $M_{s} \subseteq \mathbb{T}^{2} \backslash\left\{z_{0}, \ldots, z_{s}\right\}$ is compact and therefore $\Phi_{s}^{-1}$ is uniformly continuous on $M_{s}$. Hence there is $\eta_{s}>0$ such that $\left|\Phi_{s}^{-1}(x)-\Phi_{s}^{-1}(y)\right|<1 / 2^{s}$ whenever $x, y \in M_{s}$ and $|x-y| \leq \eta_{s}$.

Now we are ready to formulate the last conditions for the squares $A_{n}$. First of all let $A_{n} \subseteq A_{n}^{*}$ (with the same centre as $A_{n}^{*}$ ). Then we have

$$
T\left(A_{s+3}\right) \cup T^{-1}\left(A_{s+3}\right) \subseteq M_{s} .
$$

Finally let

$$
\left|A_{n}\right|<\min \left\{\eta_{n-1}, \ldots, \eta_{n-5}\right\} .
$$

We have finished the definition of the sequence $\left(A_{n}\right)_{n=0}^{\infty}$.

(I) Definition of maps $\sigma, \tau: \mathbb{N} \rightarrow \mathbb{N}$ and $\Phi_{\sigma, n}, \Phi_{\tau, n}: \mathbb{T}^{2} \rightarrow \mathbb{T}^{2}$. Let $\sigma, \tau: \mathbb{N} \rightarrow \mathbb{N}$ be bijective maps such that $T\left(z_{n}\right)=z_{\sigma(n)}$ and $T^{-1}\left(z_{n}\right)=z_{\tau(n)}$. So $\tau \circ \sigma=\sigma \circ \tau=\mathrm{id}$ on $\mathbb{N}$. Then $0<|\sigma(n)-n| \leq 2$ and $0<|\tau(n)-n| \leq 2$ for all $n=0,1,2, \ldots$.

Let $\Phi_{\sigma, n}=\Lambda_{\sigma(n)} \circ \ldots \circ \Lambda_{\sigma(1)} \circ \Lambda_{\sigma(0)}$ and $\Phi_{\tau, n}=\Lambda_{\tau(n)} \circ \ldots \circ \Lambda_{\tau(1)} \circ \Lambda_{\tau(0)}$. We claim that for all $n \geq 2$,

$$
\Phi_{\sigma, n}=\Lambda_{2[n / 2]+2} \circ \Lambda_{2[n / 2]} \circ \Phi_{n-2}
$$

and

$$
\Phi_{\tau, n}=\Lambda_{2[(n-1) / 2]+3} \circ \Lambda_{2[(n-1) / 2]+1} \circ \Phi_{n-2} .
$$

The formulas can be proved by induction, using the commutativity property mentioned in (E) and distinguishing whether $n$ is even or odd.

(J) Uniform convergence of $\Phi_{n}, \Phi_{\sigma, n}$ and $\Phi_{\tau, n}$ to the same limit $\Phi$. We have $\left\|\Phi_{n+1}-\Phi_{n}\right\|=\left\|\Lambda_{n+1} \circ \Phi_{n}-\mathrm{id} \circ \Phi_{n}\right\|=\left\|\Lambda_{n+1}-\mathrm{id}\right\|<1 / 2^{n+1}$ by $(\mathrm{F})$. Hence $\Phi_{n}$ uniformly converges on $\mathbb{T}^{2}$ to some continuous map $\Phi$.

Further, $\left\|\Phi_{\sigma, n}-\Phi\right\| \leq\left\|\Phi_{\sigma, n}-\Phi_{n-2}\right\|+\left\|\Phi_{n-2}-\Phi\right\|$. The second term on the right-hand side tends to zero and by (3) the first term equals $\| \Lambda_{2[n / 2]+2} \circ$ $\Lambda_{2[n / 2]}-\mathrm{id} \| \leq \max \left\{\left|A_{2[n / 2]+2}\right|,\left|A_{2[n / 2]}\right|\right\}$, which by $(\mathrm{F})$ tends to zero.

Similarly one can show that $\Phi_{\tau, n}$ also converges to $\Phi$.

(K) Bijectivity of $\left.\Phi\right|_{X}: X \rightarrow X$. The map $\left.\Phi\right|_{X}$ is surjective, being the uniform limit of surjective maps $\left.\Phi_{n}\right|_{X}$. Suppose that $\Phi(x)=\Phi(y)$ for some 
$x, y \in X, x \neq y$. Take $n$ with $|x-y| \geq 1 / n$. Then by (G), for $m \geq n$ at least one of the points $\Phi_{m}(x), \Phi_{m}(y)$, say $\Phi_{m}(y)$, does not belong to the square $\left(a_{m+1}+1\right) A_{m+1}$. Now we distinguish two cases.

CASE 1. If $\Phi_{m}(x) \in A_{m+1}$, then $\Phi_{m+1}(x) \in A_{m+1}$ and therefore $\left|\Phi_{m+1}(x)-\Phi_{m}(x)\right| \leq\left|A_{m+1}\right|$. Further, $\left|\Phi_{m}(x)-\Phi_{m}(y)\right| \geq \frac{1}{2} a_{m+1}\left|A_{m+1}\right|$. So, taking into account that $\Phi_{m+1}(y)=\Phi_{m}(y)$, we get

$$
\begin{aligned}
\left|\Phi_{m+1}(x)-\Phi_{m+1}(y)\right| & \geq\left|\Phi_{m}(x)-\Phi_{m}(y)\right|-\left|\Phi_{m+1}(x)-\Phi_{m}(x)\right| \\
& \geq\left|\Phi_{m}(x)-\Phi_{m}(y)\right|\left(1-2 / a_{m+1}\right) .
\end{aligned}
$$

CASE 2. If $\Phi_{m}(x) \notin A_{m+1}$ then we have $\Phi_{m+1}(x)=\Phi_{m}(x)$ and similarly for $y$, hence

$$
\begin{aligned}
\left|\Phi_{m+1}(x)-\Phi_{m+1}(y)\right| & =\left|\Phi_{m}(x)-\Phi_{m}(y)\right| \\
& >\left|\Phi_{m}(x)-\Phi_{m}(y)\right|\left(1-2 / a_{m+1}\right) .
\end{aligned}
$$

In any case,

$$
\begin{aligned}
0 & =|\Phi(x)-\Phi(y)|=\lim _{m \rightarrow \infty}\left|\Phi_{m}(x)-\Phi_{m}(y)\right| \\
& \geq \prod_{r=n+1}^{\infty}\left(1-2 / a_{r}\right)\left|\Phi_{n}(x)-\Phi_{n}(y)\right|,
\end{aligned}
$$

whence $\Phi_{n}(x)=\Phi_{n}(y)$, contrary to $\left.\Phi_{n}\right|_{X}$ being a bijection.

Below in (L), (M) and (N), $A$ and $B$ will be squares with centres $z_{A}, z_{B}$ and we will assume that $T\left(z_{A}\right)=z_{B}$.

(L) Uniform continuity of $L_{A B}=\Lambda_{B}^{-1} \circ T \circ \Lambda_{A}$, where $A$ is a nondegenerate square, on $\mathbb{T}^{2} \backslash K_{z_{A}}$ (even on $\mathbb{T}^{2} \backslash I_{z_{A}}$ ). On $\mathbb{T}^{2} \backslash I_{z_{A}}, L_{A B}$ is obviously defined and continuous.

To prove the uniform continuity of $L_{A B}$ on $\mathbb{T}^{2} \backslash I_{z_{A}}$ it is sufficient to prove that $L_{A B}$ can be continuously extended to the whole torus. The set $M=A \cap\left(T \circ \Lambda_{A}\right)^{-1}(B)$ contains an open neighbourhood of $I_{z_{A}}$ and if $z_{A}+(t, s) \in M \backslash I_{z_{A}}$, then by (0) we have

$$
L_{A B}\left(z_{A}+(t, s)\right)=z_{B}+\left(t, h_{\eta} \circ h_{\delta}^{-1}(s)\right)
$$

where $|A|=2 \delta,|B|=2 \eta$. If we define $L_{A B}$ on the whole set $M$ (including $I_{z_{A}}$ ) by the formula (5), then it is obviously continuous on $M$. Thus $L_{A B}$, being continuous both on $\mathbb{T}^{2} \backslash I_{z_{A}}$ and on a neighbourhood of $I_{z_{A}}$, is continuous on $\mathbb{T}^{2}$ and the result follows.

(M) Uniform continuity of $L_{A B}$ on $\mathbb{T}^{2}$ if both $A$ and $B$ are degenerate squares. This is obvious, since in this case $L_{A B}=T$.

(N) Nonuniform continuity of $L_{A B}$ on $\mathbb{T}^{2} \backslash\left\{z_{A}\right\}$ if $A$ is a degenerate and $B$ a nondegenerate square. In this case we have $L_{A B}=\Lambda_{B}^{-1} \circ T \circ \mathrm{id}$ and the result follows from the nonuniform continuity of $\Lambda_{B}^{-1}$. 
(O) Uniform continuity of the map $S_{n}=\Phi_{\sigma, n}^{-1} \circ T \circ \Phi_{n}$ defined on the set $X$. Since in this part we work on $X$, all the sets should be considered to be intersected with $X$ even if we do not state it explicitly.

We prove this by induction. We have $S_{0}=\Lambda_{2}^{-1} \circ T \circ \Lambda_{0}$ and since $A_{0}$ is a nondegenerate square and $A_{2}$ is degenerate, it suffices to use (L).

Assume inductively that $S_{n}$ is uniformly continuous on $X$. Since $S_{n}=$ $\Phi_{\sigma, n}^{-1} \circ T \circ \Phi_{n}$ and $S_{n+1}=\Phi_{\sigma, n}^{-1} \circ L_{n+1, \sigma(n+1)} \circ \Phi_{n}$ where $L_{n+1, \sigma(n+1)}=$ $\Lambda_{\sigma(n+1)}^{-1} \circ T \circ \Lambda_{n+1}$, we have

$$
\begin{aligned}
\left\{z: S_{n+1}(z)\right. & \left.\neq S_{n}(z)\right\} \\
& \subseteq\left\{z: \Phi_{n}(z) \in A_{n+1}\right\} \cup\left\{z: T \circ \Lambda_{n+1} \circ \Phi_{n}(z) \in A_{\sigma(n+1)}\right\} .
\end{aligned}
$$

The maps $\Lambda_{i}, \Phi_{i}$ do not change the first coordinates of the points and so

$$
\begin{aligned}
\{z: & \left.S_{n+1}(z) \neq S_{n}(z)\right\} \\
& \subseteq \pi\left(A_{n+1}\right) \times K \cup\left[x_{n+1}-\left|A_{\sigma(n+1)}\right| / 2, x_{n+1}+\left|A_{\sigma(n+1)}\right| / 2\right] \times K \\
& =\left[x_{n+1}-\alpha_{n+1} / 2, x_{n+1}+\alpha_{n+1} / 2\right] \times K=D_{n+1}
\end{aligned}
$$

where $\alpha_{n+1}=\max \left\{\left|A_{n+1}\right|,\left|A_{\sigma(n+1)}\right|\right\}$.

The set $D_{n+1}$ is invariant under all $\Lambda_{m}$ and is mapped to

$$
\left[x_{\sigma(n+1)}-\alpha_{n+1} / 2, x_{\sigma(n+1)}+\alpha_{n+1} / 2\right] \times K=T\left(D_{n+1}\right)
$$

by $L_{n+1, \sigma(n+1)}$ and $T\left(D_{n+1}\right)$ is invariant under all $\Lambda_{m}$.

Since $S_{n+1}=S_{n}$ on (at least) $X \backslash D_{n+1}$ and $S_{n}$ is uniformly continuous on $X$, to finish the proof it suffices to show that $S_{n+1}$ is uniformly continuous on $D_{n+1} \backslash K_{z_{n+1}}$. The map $\Phi_{n}$ is uniformly continuous on $D_{n+1}$. The compact set $T\left(D_{n+1}\right)$ does not intersect $K_{x_{i}}$ for $i \in\{\sigma(0), \sigma(1), \ldots, \sigma(n)\}$, therefore $\Phi_{\sigma, n}^{-1}$ is uniformly continuous on $T\left(D_{n+1}\right)$.

We now show that $L_{n+1, \sigma(n+1)}$ is uniformly continuous on $D_{n+1} \backslash K_{z_{n+1}}$. But this restriction is uniformly continuous if $A_{n+1}$ is nondegenerate (by (L)) or $A_{\sigma(n+1)}$ is degenerate, and is not uniformly continuous if $A_{n+1}$ is degenerate but $A_{\sigma(n+1)}$ nondegenerate. Since by the definition of the sequence $\left(A_{i}\right)_{i=0}^{\infty}$ this last case cannot occur, $L_{n+1, \sigma(n+1)}$ is uniformly continuous on $D_{n+1} \backslash K_{z_{n+1}}$.

Now the uniform continuity of

$$
S_{n+1}=\Phi_{\sigma, n}^{-1} \circ L_{n+1, \sigma(n+1)} \circ \Phi_{n}
$$

on the set $D_{n+1} \backslash K_{z_{n+1}}$ follows from the facts that $\Phi_{n}$ is uniformly continuous on $D_{n+1} \backslash K_{z_{n+1}}, \Phi_{n}\left(D_{n+1} \backslash K_{z_{n+1}}\right)=D_{n+1} \backslash K_{z_{n+1}}, L_{n+1, \sigma(n+1)}$ is uniformly continuous on $D_{n+1} \backslash K_{z_{n+1}}, L_{n+1, \sigma(n+1)}\left(D_{n+1} \backslash K_{z_{n+1}}\right) \subseteq T\left(D_{n+1}\right)$ and $\Phi_{\sigma, n}^{-1}$ is uniformly continuous on $T\left(D_{n+1}\right)$. 
(P) Nonuniform continuity of the map $S_{n}^{*}=\Phi_{\tau, n}^{-1} \circ T^{-1} \circ \Phi_{n}$ defined on the set $X$ for $n \geq 2$. As in the previous part, all the sets should be considered to be intersected with $X$.

First notice that $S_{0}^{*}$ and $S_{1}^{*}$ are uniformly continuous on $X$ by the same argument as for $S_{n}, n \in \mathbb{N}$. Roughly speaking, the reason why $S_{n}^{*}, n \geq 2$, are not uniformly continuous on $X$ is that the map $L_{2,0}^{*}=\Lambda_{0}^{-1} \circ T^{-1} \circ$ $\Lambda_{2}=\Lambda_{0}^{-1} \circ T^{-1} \circ \mathrm{id}$ is not uniformly continuous (this map is defined on $\mathbb{T}^{2} \backslash\left\{z_{2}\right\}$, it sends the points close to $z_{2}$ to points close to $z_{0}$ and is not uniformly continuous on any neighbourhood of $z_{2}$ ). We show that this fact really implies the nonuniform continuity of $S_{n}^{*}, n \geq 2$.

Quite analogously to the case of the maps $S_{n}$ (just replace $T$ by $T^{-1}$ and $\sigma$ by $\tau$ ) we get

$$
\left\{z: S_{n+1}^{*}(z) \neq S_{n}^{*}(z)\right\} \subseteq\left[x_{n+1}-\alpha_{n+1}^{*} / 2, x_{n+1}+\alpha_{n+1}^{*} / 2\right] \times K=D_{n+1}^{*}
$$

where $\alpha_{n+1}^{*}=\max \left\{\left|A_{n+1}\right|,\left|A_{\tau(n+1)}\right|\right\}$. The set $D_{n+1}^{*}$ is invariant under all $\Lambda_{m}$ and is mapped to $\left[x_{\tau(n+1)}-\alpha_{n+1}^{*} / 2, x_{\tau(n+1)}+\alpha_{n+1}^{*} / 2\right] \times K=T^{-1}\left(D_{n+1}^{*}\right)$ by $L_{n+1, \tau(n+1)}^{*}=\Lambda_{\tau, n+1}^{-1} \circ T^{-1} \circ \Lambda_{n+1}$ and $T^{-1}\left(D_{n+1}^{*}\right)$ is invariant under all $\Lambda_{m}$.

The map $S_{1}^{*}$ is uniformly continuous on $X$ and $S_{2}^{*}=S_{1}^{*}$ on (at least) $X \backslash D_{2}^{*}$, so $S_{2}^{*}$ is uniformly continuous on $X \backslash D_{2}^{*}$. But $S_{2}^{*}=\Phi_{\tau, 1}^{-1} \circ L_{2,0}^{*} \circ \Phi_{1}$ is not uniformly continuous on $D_{2}^{*}$ because $\Phi_{1}=$ id on $D_{2}^{*}, L_{2,0}^{*}$ is not uniformly continuous on $D_{2}^{*}, L_{2,0}^{*}\left(D_{2}^{*}\right)=T^{-1}\left(D_{2}^{*}\right)$ and $\Phi_{\tau, 1}^{-1}=$ id on $T^{-1}\left(D_{2}^{*}\right)$. Thus we have proved that $S_{2}^{*}$ is not uniformly continuous on $X \supseteq X \backslash D_{3}^{*} \supseteq D_{2}^{*}$.

Assume that $S_{n}^{*}$ is not uniformly continuous on $X \backslash D_{n+1}^{*}$. We have $S_{n+1}^{*}=S_{n}^{*}$ on $X \backslash D_{n+1}^{*}$, therefore $S_{n+1}^{*}$ is not uniformly continuous on $X \supseteq X \backslash D_{n+1}^{*}$.

(Q) The sequences $\left(S_{n}\right)_{n=0}^{\infty}$ and $\left(S_{n}^{*}\right)_{n=0}^{\infty}$ are Cauchy sequences on $X$. By (3) for $n \geq 2$ we have

$$
S_{n}=\Phi_{\sigma, n}^{-1} \circ T \circ \Phi_{n}=\Phi_{n-2}^{-1} \circ \Lambda_{2[n / 2]}^{-1} \circ \Lambda_{2[n / 2]+2}^{-1} \circ T \circ \Lambda_{n} \circ \Lambda_{n-1} \circ \Phi_{n-2}
$$

and

$$
\begin{aligned}
S_{n+1} & =\Phi_{\sigma, n}^{-1} \circ \Lambda_{\sigma(n+1)}^{-1} \circ T \circ \Lambda_{n+1} \circ \Phi_{n} \\
& =\Phi_{n-2}^{-1} \circ \Lambda_{2[n / 2]}^{-1} \circ \Lambda_{2[n / 2]+2}^{-1} \circ \Lambda_{\sigma(n+1)}^{-1} \circ T \circ \Lambda_{n+1} \circ \Lambda_{n} \circ \Lambda_{n-1} \circ \Phi_{n-2} .
\end{aligned}
$$

Since $S_{n}=S_{n+1}$ on $X \backslash D_{n+1}$, we need only estimate $\left\|S_{n+1}-S_{n}\right\|$ on $D_{n+1}$.

First notice that $n+1 \notin\{n-1, n\}$ and so $A_{n-1}$ and $A_{n}$ are disjoint from $D_{n+1}$. Further, both for even and odd $n$ (note that $n \geq 2$ ), $\sigma(n+1) \notin$ $\{2[n / 2], 2[n / 2]+2\}$ and so $A_{2[n / 2]}$ and $A_{2[n / 2]+2}$ are disjoint from $T\left(D_{n+1}\right)$. Finally, recall that $\Phi_{n-2}$ as well as all $\Lambda_{i}$ do not change the first coordinates of points. As a result of all these facts we get

$$
S_{n}=\Phi_{n-2}^{-1} \circ T \circ \Phi_{n-2} \quad \text { on } D_{n+1}
$$


and

$$
S_{n+1}=\Phi_{n-2}^{-1} \circ \Lambda_{\sigma(n+1)}^{-1} \circ T \circ \Lambda_{n+1} \circ \Phi_{n-2} \quad \text { on } D_{n+1} .
$$

Hence, on $X$ we have

$$
\begin{aligned}
\| S_{n+1}- & S_{n} \| \\
= & \| \Phi_{n-2}^{-1} \circ \Lambda_{\sigma(n+1)}^{-1} \circ T \circ \Lambda_{n+1} \circ \Phi_{n-2}-\Phi_{n-2}^{-1} \circ T \circ \Lambda_{n+1} \circ \Phi_{n-2} \\
& +\Phi_{n-2}^{-1} \circ T \circ \Lambda_{n+1} \circ \Phi_{n-2}-\Phi_{n-2}^{-1} \circ T \circ \Phi_{n-2} \| \\
\leq & \left\|\Phi_{n-2}^{-1} \circ \Lambda_{\sigma(n+1)}^{-1}-\Phi_{n-2}^{-1}\right\|+\left\|\Phi_{n-2}^{-1} \circ T \circ \Lambda_{n+1}-\Phi_{n-2}^{-1} \circ T\right\| \\
\leq & \max _{n-1 \leq r \leq n+3}\left\|\Phi_{n-2}^{-1} \circ \Lambda_{r}^{-1}-\Phi_{n-2}^{-1}\right\|+\left\|\Phi_{n-2}^{-1} \circ T \circ \Lambda_{n+1}-\Phi_{n-2}^{-1} \circ T\right\|
\end{aligned}
$$

(in the last inequality we used the fact that $|\sigma(n)-n| \leq 2$ and so $\sigma(n+1) \in$

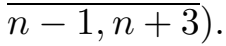

By $(\mathrm{E})$, if $n-1 \leq r \leq n+3, x \in X$ and $\Lambda_{r}^{-1}(x) \neq x$ (hence, $x \in A_{r}$ ), then $x, \Lambda_{r}^{-1}(x) \in A_{r} \cap M_{n-2}$ (we used the fact that by $(\mathrm{H})$ for any $k, M_{k}$ contains the squares $\left.A_{k+1}, \ldots, A_{k+5}\right)$.

By (2), $\left|\Lambda_{r}^{-1}(x)-x\right|<\left|A_{r}\right|<\eta_{n-2}$. It follows that

$$
\max _{n-1 \leq r \leq n+3}\left\|\Phi_{n-2}^{-1} \circ \Lambda_{r}^{-1}-\Phi_{n-2}^{-1}\right\|<1 / 2^{n-2} .
$$

If $T \circ \Lambda_{n+1}(x) \neq T(x)$ then $\Lambda_{n+1}(x) \neq x$ and so $x, \Lambda_{n+1}(x) \in A_{n+1}$. Then by (2), $\left|T \circ \Lambda_{n+1}(x)-T(x)\right|=\left|\Lambda_{n+1}(x)-x\right| \leq\left|A_{n+1}\right|<\eta_{n-2}$. By (1), $T\left(A_{n+1}\right) \subseteq M_{n-2}$, whence $T(x), T \circ \Lambda_{n+1}(x) \in M_{n-2}$ and taking into account the above inequality, we get

$$
\left\|\Phi_{n-2}^{-1} \circ T \circ \Lambda_{n+1}-\Phi_{n-2}^{-1} \circ T\right\|<1 / 2^{n-2} .
$$

From (10) and (11) we have $\left\|S_{n+1}-S_{n}\right\|<1 / 2^{n-3}$ and so $\left(S_{n}\right)_{n=0}^{\infty}$ is a Cauchy sequence. Denote its uniform limit on $X$ by $S$.

The proof for $\left(S_{n}^{*}\right)_{n=0}^{\infty}$ is analogous, the difference being that one has $\tau$ and $T^{-1}$ instead of $\sigma$ and $T$, respectively. Denote the uniform limit of $S_{n}^{*}$ on $X$ by $S^{*}$.

(R) Definition of the continuous surjective map $\widetilde{S}$ on $\mathbb{T}^{2}$. Since the map $S_{n}$ is uniformly continuous on the dense subset $X$ of $\mathbb{T}^{2}$, there is a unique continuous extension $\widetilde{S}_{n}$ of $S_{n}$ to $\mathbb{T}^{2}$. Since $\left(S_{n}\right)_{n=0}^{\infty}$ is a Cauchy sequence, obviously $\left(\widetilde{S}_{n}\right)_{n=0}^{\infty}$ is also Cauchy and so has a uniform limit $\widetilde{S}$. Clearly, $\left.\widetilde{S}\right|_{X}=S$. Since $S_{n}(X)=X$ for any $n$, we have $S(X)=X$ and so $\widetilde{S}\left(\mathbb{T}^{2}\right)=\mathbb{T}^{2}$.

(S) Noninvertibility of $\widetilde{S}$. Recall that, by definition, $\Phi_{\sigma, n} \circ S_{n}=T \circ \Phi_{n}$ and $\Phi_{\tau, n} \circ S_{n}^{*}=T^{-1} \circ \Phi_{n}$ on the set $X$. Further, $\Phi_{\sigma, n}, \Phi_{\tau, n}$ and $\Phi_{n}$ uniformly converge to $\Phi$ on the torus, and the map $\Phi$ as well as all the maps $\Phi_{\sigma, n}, \Phi_{\tau, n}$, $\Phi_{n}, n=1,2, \ldots$, are uniformly continuous. It follows that $\Phi \circ S=T \circ \Phi$ and $\Phi \circ S^{*}=T^{-1} \circ \Phi$ on $X$. (We used the fact that $f_{n} \circ g_{n}$ uniformly converge 
to $f \circ g$ whenever $g_{n}$ uniformly converge to $g, f_{n}$ uniformly converge to $f$ and the maps $f_{n}, n=1,2, \ldots$, are equicontinuous - which is the case when $f_{n}, n=1,2, \ldots$, and $f$ are uniformly continuous and $f_{n}$ uniformly converge to $f$.)

Since $\Phi$ is bijective (see (K)), we get $S=\Phi^{-1} \circ T \circ \Phi$ and $S^{*}=\Phi^{-1} \circ T^{-1} \circ \Phi$ on $X$. Hence $S^{*}=S^{-1}$ on $X$. Since $\left.\widetilde{S}\right|_{X}=S$ and $\widetilde{S}(X)=X$ we have $\left(\left.\widetilde{S}\right|_{X}\right)^{-1}=S^{*}$. But $S^{*}$ is not uniformly continuous on $X$ (by $\left.(\mathrm{P})\right)$, therefore $\widetilde{S}$ does not have an inverse on $\mathbb{T}^{2}$.

(T) Minimality of $\widetilde{S}$. Take any nonempty closed set $M \subseteq \mathbb{T}^{2}$ with $\widetilde{S}(M) \subseteq M$; we need to show that $M=\mathbb{T}^{2}$. Since $\widetilde{S}$ is an extension of $T$, we have $T(\Phi(M))=\Phi(\widetilde{S}(M)) \subseteq \Phi(M)$ and by minimality of $T$ we get $\Phi(M)=\mathbb{T}^{2}$. Recall that $\Phi=\lim _{n \rightarrow \infty} \Phi_{n}$ and the maps $\Phi_{n}$ do not change the first coordinate of points. Therefore also $\Phi$ has this property, i.e., $\Phi(x, y)=(x, \phi(x, y))$. Hence $\Phi(X)=X$ and $\Phi\left(\mathbb{T}^{2} \backslash X\right)=\mathbb{T}^{2} \backslash X$. It follows that $\Phi(M \cap X)=X$. Since $\left.\Phi\right|_{X}: X \rightarrow X$ is a bijection (see $(\mathrm{K})$ ), $M \cap X=X$. Now use the density of $X$ and the closedness of $M$ in $\mathbb{T}^{2}$ to conclude that $M=\mathbb{T}^{2}$.

Added in proof (March 2001). After submitting the paper the authors (S. Kolyada and L'. Snoha) and H. Bruin found a compact metric space which admits a minimal map but does not admit any minimal homeomorphism (cf. Introduction).

\section{References}

[Al] P. S. Aleksandrov, Introduction to Set Theory and to General Topology, Nauka, Moscow, 1977 (in Russian); German translation: P. S. Alexandroff, Einführung in die Mengenlehre und in die allgemeine Topologie, Deutscher Verlag der Wiss., Berlin, 1984.

[Au] J. Auslander, Minimal Flows and their Extensions, North-Holland Math. Stud. 153, Elsevier, Amsterdam, 1988.

[AG] J. Auslander and W. H. Gottschalk (eds.), Topological Dynamics, An International Symposium, W. A. Benjamin, New York, 1968.

[AK] J. Auslander and Y. Katznelson, Continuous maps of the circle without periodic points, Israel J. Math. 32 (1979), 375-381.

[AY] J. Auslander and J. A. Yorke, Interval maps, factors of maps, and chaos, Tôhoku Math. J. 32 (1980), 177-188.

[BL] A. M. Blokh and M. Lyubich, Measurable dynamics of S-unimodal maps, Ann. Sci. Ecole Norm. Sup. 24 (1991), 545-573.

[Br] I. U. Bronšteĭn, Extensions of Minimal Transformation Groups, Sijthoff and Noordhoff, 1979.

[BKNS] H. Bruin, G. Keller, T. Nowicki and S. van Strien, Wild Cantor attractors exist, Ann. of Math. 143 (1996), 97-130.

[BKP] H. Bruin, G. Keller and M. St. Pierre, Adding machines and wild attractors, Ergodic Theory Dynam. Systems 17 (1997), 1267-1287. 
[F] Z. Frolík, Remarks concerning the invariance of Baire spaces under mappings, Czechoslovak Math. J. 11 (86) (1961), 381-385.

[FW] H. Furstenberg and B. Weiss, On almost 1-1 extensions, Israel J. Math. 65 (1989), 311-322.

[G] W. H. Gottschalk, Orbit-closure decompositions and almost periodic properties, Bull. Amer. Math. Soc. 50 (1944), 915-919.

[HK] T. Homma and S. Kinoshita, On the regularity of homeomorphisms of $E^{n}$, J. Math. Soc. Japan 5 (1953), 365-371.

[KS] S. Kolyada and L'. Snoha, Some aspects of topological transitivity - a survey, Grazer Math. Ber. 334 (1997), 3-35.

[K1] K. Kuratowski, Topology, Vol. I, Academic Press and PWN, New York and Warszawa, 1966.

[LCY] P. Le Calvez and J. Yoccoz, Un théorème d'indice pour homéomorphismes du plan au voisinage d'un point fixe, Ann. of Math. 146 (1997), 241-293.

[LMa] D. Lind and B. Marcus, Symbolic Dynamics and Coding, Cambridge Univ. Press, Cambridge, 1995.

[LM] M. Lyubich and J. Milnor, The unimodal Fibonacci map, J. Amer. Math. Soc. 6 (1993), 425-457.

[M] P. Maličký, Backward orbits of transitive maps, preprint.

[MW] J. van Mill and R. G. Woods, Perfect images of zero-dimensional separable metric spaces, Canad. Math. Bull. 25 (1982), 41-47.

[O] J. C. Oxtoby, Measure and Category, Springer, New York, 1971.

[PW] J. R. Porter and R. G. Woods, Extensions and Absolutes of Hausdorff Spaces, Springer, New York, 1988.

[R] M. Rees, A point distal transformation of the torus, Israel J. Math. 32 (1979), 201-208.

[RS] J. H. Roberts and N. E. Steenrod, Monotone transformations of the 2-dimensional manifolds, Ann. of Math. 39 (1938), 851-862.

[S] S. Silverman, On maps with dense orbits and the definition of chaos, Rocky Mountain J. Math. 22 (1992), 353-375.

[deV] J. de Vries, Elements of Topological Dynamics, Math. Appl. 257, Kluwer, Dordrecht, 1993.

[W] P. Walters, An Introduction to Ergodic Theory, Grad. Texts in Math. 79, Springer, Berlin, 1982.

Institute of Mathematics

Ukrainian Academy of Sciences

Tereshchenkivs'ka 3

252601 Kiev, Ukraine

E-mail: skolyada@imath.kiev.ua

Departamento de Matemáticas

Facultad de Ciencias

Universidad de Chile

Las Palmeras 3425

Santiago, Chile

E-mail: trofimch@abello.dic.uchile.cl
Department of Mathematics Faculty of Natural Sciences Matej Bel University Tajovského 40 97401 Banská Bystrica, Slovakia E-mail: snoha@fpv.umb.sk

Received 30 June 2000; in revised form 5 October 2000 